\title{
Perception of pictorial human faces by baboons: Effects of stimulus orientation on discrimination performance
}

\author{
JULIE MARTIN-MALIVEL and JOËL FAGOT \\ CNRS, Center for Research in Cognitive Neurosciences, Marseille, France
}

\begin{abstract}
The effect of stimulus rotation was assessed in four Guinea baboons (Papio papio), using pictures of familiar human faces presented in a computerized go/no-go task. In Experiment 1, 2 baboons were initially trained to discriminate upright faces, and 2 others were trained to discriminate upside-down faces. For the two groups, postlearning discrimination was impaired when the training faces were rotated $180^{\circ}$. In Experiment 2, upright and upside-down priming faces appeared prior to the display of target faces. For the two groups, response times were faster when the prime and the target faces had the same orientations than when they were depicted under different orientations. Finally, Experiments 3 and 4 identified variations in facial contours as the most salient discriminative cue controlling performance in 2 baboons. Altogether, our results provide no evidence that the baboons processed the pictures as representations of faces. It is suggested that the effect of rotation derived from the encoding of the pictorial faces as meaningless mono-oriented shapes, rather than as natural human faces.
\end{abstract}

In humans, turning faces upside down has a detrimental effect on the capacity to identify individuals, to perceive their emotional expressions, and to discriminate or memorize the faces. This phenomenon, coined the inversion effect, is stronger for faces than for other classes of mono-oriented objects, such as cars or houses (see Farah, Wilson, Drain, \& Tanaka, 1998; Valentine, 1988), therefore suggesting that face perception involves processes different from those underlying the recognition of other classes of objects (e.g., Yin, 1969).

The effect of stimulus inversion has been intensively studied in humans (for a review, see Farah et al., 1998), but to a more limited extent in animals (e.g., Bruce, 1982; Dittrich, 1990; Overman \& Doty, 1982; Rosenfeld \& van Hoesen, 1979; Tomonaga, Itakura, \& Matsuzawa, 1993; Vermeire \& Hamilton, 1998). From a comparative perspective, however, it is critical to verify whether animals, especially nonhuman primates, that have a visual system similar to that of human primates are also sensitive to the inversion of facial stimuli. Thus, observing an inversion effect in these species would suggest that human and nonhuman primates share similar neural and psychological mechanisms for face processing, despite evolutionary differences, and may also provide some insights for explaining them. From a more practical perspective, comparative studies on the effect of face inversion in animals can provide additional validations for the use of animal species as experimental models for studying perceptual/

We thank B. Arnaud, M. Chiambretto, and R. Fayolle for technical assistance. Correspondence should be addressed to J. Fagot, Center for Research in Cognitive Neurosciences, CNRS, 31 chemin Joseph-Aiguier, 13402 Marseille, Cedex 20, France (e-mail: fagot@lnf.cnrs-mrs.fr). cognitive processing in humans and the underlying brain mechanisms of such processing.

Behavioral investigations of the effect of inversion in monkeys and apes have provided inconsistent results (for a recent review, see Pascalis, Petit, Kim, \& Campbell, 1999). Thus, several studies have reported that inversion of facial stimuli $180^{\circ}$ has little effect on recognition performance, if any. For instance, Rosenfeld and van Hoesen (1979) found that inversion of the face of conspecifics did not disrupt the performance of rhesus macaques ( $\mathrm{Ma}$ caca mulatta) in a forced-choice discrimination task. Using pictures of conspecifics, Bruce (1982) also found no effect of stimulus orientation in cynomolgus macaques (Macaca fascicularis) tested in a simultaneous discrimination task. Dittrich (1990) showed that inversion of schematic monkey faces with different emotional expressions did not hamper discrimination performance in the same species. In 1 chimpanzee (Pan troglodytes), Tomonaga et al. (1993) found no clear-cut effect of stimulus inversion in a face discrimination task involving familiar faces of humans and chimpanzees.

By contrast, several other behavioral studies have successfully demonstrated effects of stimulus inversion in nonhuman primates, although these effects do not always parallel the inversion effect reported in the literature on humans. For instance, by way of a habituationdishabituation procedure, Swartz (1982) observed that 3month-old pigtail macaques (Macaca nemestrina) discriminate faces of adult macaques when they are presented right side up, but not when they are presented upside down. Overman and Doty (1982) found a decline of performance when pigtailed macaques viewed inverted human and rhesus faces, but no effect of stimulus inversion for scenes. The effect of face inversion was also re- 
ported in a squirrel monkey (Saimiri sciureus), but this effect appeared for human faces only, and not for monkey faces or scenes (Phelps \& Roberts, 1994). Wright and Roberts (1996) reported similar results on rhesus monkeys. Parr, Dove, and Hopkins (1998) tested the discrimination of various kinds of upright and upside-down visual objects by chimpanzees. Discrimination was better when the human and chimpanzee faces were presented right side up, as compared with upside-down presentations, whereas no significant difference appeared for capuchin faces and automobiles. Tests for the effect of face inversion have also been conducted in the context of electrophysiological studies devoted to the investigation of the so-called face neurons, whose activity is tuned to the presentation of facial stimuli. Thus, Perrett et al. (1984) reported a decline in the activity of face neurons with inverted faces, as compared with right side up faces. However, other studies failed to replicate this finding (e.g., Hasselmo, Rolls, Baylis, \& Nalwa, 1989).

In brief, this literature indicates that rotating facial pictures $180^{\circ}$ has inconsistent effects in nonhuman primates. One possible explanation for these inconsistencies between studies might be that animals did not systematically recognize faces in pictures. Unfortunately, the above studies provide little information (if any) on that question and do not demonstrate that the visual stimuli were perceived and processed as facial stimuli by the animals, instead of being perceived as nonmeaningful stimuli. Interestingly enough, recent review papers on the perception of pictures (Fagot, 2000; Fagot, Martin-Malivel, \& Dépy, 1999), including pictures of faces (Huber, 1999; Pascalis et al., 1999), reveal that birds and even nonhuman primates do not always process pictures as equivalent to the real objects they are supposed to depict. Determining whether animal subjects are capable of recognizing faces in pictures appears to be a prerequisite for interpreting discrimination studies involving facial pictures.

Considering the above remarks, the present research had two main objectives. The first objective was to determine the possible effects of stimulus rotation on recognition performance when baboons discriminate facial pictures of familiar humans. That goal was achieved in Experiment 1 by using a go/no-go procedure and was completed in Experiment 2 by using a priming procedure adapted from the initial go/no-go task. Our second objective was to determine how baboons encoded the pictures of human faces in our testing conditions. That goal was achieved in Experiments 3 and 4 by selectively altering some of the facial features, such as their contour or their internal parts.

\section{EXPERIMENT 1}

Two groups of baboons were used in Experiment 1 . The first one will hereafter be referred to as the canonical group. It comprised baboons initially trained to discriminate pairs of pictorial human faces presented in a canonical upright orientation. After training, these animals were tested with novel pictures of the same two faces that were rotated $180^{\circ}$ or presented right side up. The second group will be referred to as the control group. Baboons from this group were initially trained to discriminate faces presented upside down, prior to being tested with upright faces. For these two groups, the effects of stimulus rotation on discrimination performance were expected to indicate to what extent the processing of the facial stimuli depended on their orientation. In addition, a direct comparison of the training performance achieved by baboons should indicate whether discrimination is facilitated when the stimuli are presented in a canonical orientation and should thus provide a first insight into whether or not baboons perceived the stimuli as representations of natural human faces. Note that we will avoid the term inversion effect in the remaining text, because it specifically refers in the literature to the effect of turning upright faces upside down. We will use instead the label rotation effect, which is defined as the effect of a $180^{\circ}$ rotation from the training orientation.

\section{Method}

Subjects. The subjects were 4 adult Guinea baboons (Papio papio), 3 males (B03, B07, and B11) and 1 female (B08), living in two social groups within the animal facility of the Center for Research in Cognitive Neurosciences, Marseille. The baboons were already familiar with the go/no-go procedure and the joystick system used in this research, owing to their previous participation in a variety of cognitive experiments (e.g., Deruelle \& Fagot, 1997; Fagot \& Deruelle, 1997). They were also tested in experiments involving various pictures of humans, including pictures of human faces, but were never presented with inverted faces (Fagot et al., 1999). The baboons were not food deprived, but they received their food ration after completion of daily training or testing sessions.

Apparatus. The baboons were tested in a cage $(68 \times 50 \times 72 \mathrm{~cm})$ facing a joystick and a 14-in. color monitor driven by a Pentium 133 PC computer. The cage was fitted with a view port, two hand ports, and a food dispenser delivering 190-mg banana-flavored food pellets within the cage when the animal responded correctly. The joystick controlled the displacements of a cursor on the monitor screen. The distance between the view port and the monitor was $49 \mathrm{~cm}$.

Stimuli. The stimuli were colored studio pictures of the faces of two familiar animal keepers that were digitized to be displayed on the monitor screen (see Figure 1). Frontal views of faces were photographed on a black background while the models wore a black scarf to mask the hair and ears. Use of the scarf was aimed at minimizing the possibility that subjects use some nonfacial features for discrimination, such as variations in haircut. The models expressed a neutral emotional state and lacked outstanding distinguishing facial features, such as glasses or beards. In order to induce some variations in the stimulus set, the faces were photographed under lighting conditions varying in the localization of the light source and its intensity. As is demonstrated in Figure 1, that procedure introduced noticeable variations between pictures-in particular, in terms of picture brightness, apparent skin texture, and location of shading area.

Altogether, the experiment involved eight different pairs of pictures, each consisting of one view of each model. Five pairs of pictures were used for training. The remaining three were used for testing. Pictures measured approximately $8 \times 6 \mathrm{~cm}$ in size on the monitor screen $\left(6.0^{\circ} \times 6.0^{\circ}\right.$ of visual angle $)$ and sustained a $640 \times$ 480 pixel definition using a palette of 256 different colors.

General Procedure. A go/no-go procedure was adopted in this task. At the beginning of each trial, a $0.5-\mathrm{cm}$ circular cursor appeared on the screen, along with a $0.5 \times 0.5 \mathrm{~cm}$ square-shaped starting stimulus located $1.5 \mathrm{~cm}$ above or below the cursor. The baboons initiated a trial by manipulating the joystick so as to place 

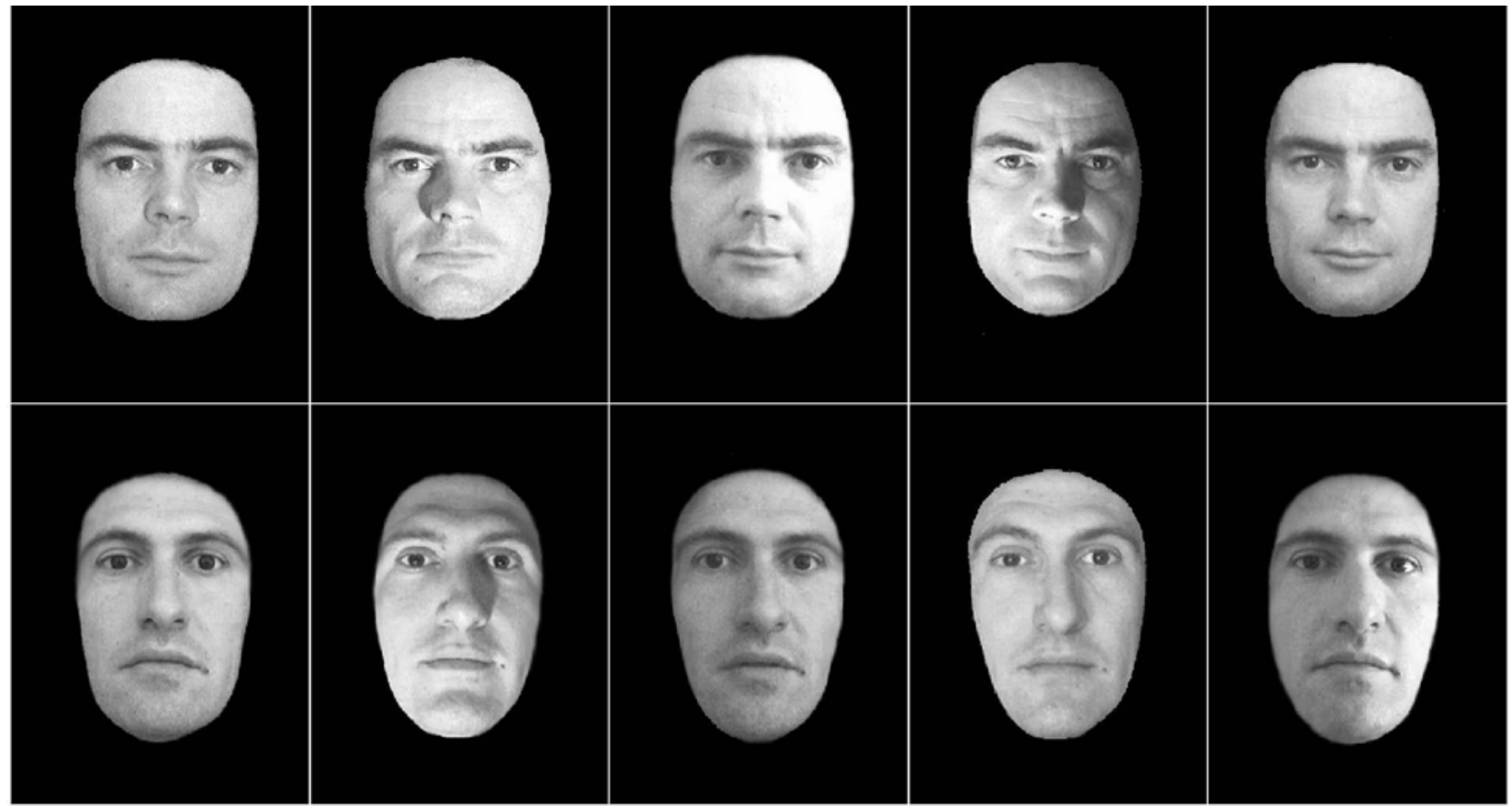

Figure 1. Illustration of the type of pictures used in Experiment 1. Note that these stimuli were presented either upright or upside down, depending on the trial type. Note also that the actual stimuli were colored pictures.

the cursor on the starting stimulus. That procedure induced eye fixation on the starting stimulus (see Wilde, Vauclair, \& Fagot, 1994) and thus controlled for the location of the gaze at the onset of the discriminative stimulus. Once done, one of two possible pictures appeared centrally on the screen. The picture could be either a no-go stimulus, corresponding to the face of one animal keeper, or a $g o$ stimulus, corresponding to the face of the other keeper. The task was to move the joystick when the go stimulus was displayed, and to refrain from moving it when the picture corresponded to the nogo stimulus. A no-go response was considered to be correct when the computer detected no joystick movement during the $3 \mathrm{sec}$ following the onset of the face. A go response was considered to be correct when the subject moved the joystick in any direction within these $3 \mathrm{sec}$. Whatever the types of trials, go or no-go, correct responses resulted in the delivery of a food pellet. The intertrial interval was set to $3 \mathrm{sec}$. Moreover, a low raucous tone and a time-out of $5 \mathrm{sec}$ followed incorrect responses. The experiment comprised five training phases, each involving a different pair of pictures, followed by a testing phase.

Training phases. Training sessions consisted of 40 go and 40 no-go trials that were presented in a pseudorandom order, with the constraint that no more than three go or no-go trials be presented in succession. The subjects from the canonical group (B03 and B07) perceived the faces upright. Those from the control group (B08 and B11) perceived them upside down. One keeper was used as the go stimulus for B03 and B08, whereas the other keeper was used as the go stimulus for B07 and B11. Each training session involved a single pair of pictures. Sessions were repeated until subjects performed at least $80 \%$ correct in two consecutive sessions, after which a novel pair of training pictures was used. The baboons thus received at least two sessions with each pair of faces.

Testing phase. The baboons from the two experimental groups received 24 test trials each. Testing involved three novel pairs of pictures (six pictures in total), each picture being displayed upright in two trials and upside down in two other trials. Therefore, only in half of the trials was the orientation of the faces the same as during training. Hereafter, these trials will be referred to as the same trials. The other half of the trials will be referred to as the different trials. The order of trials was randomized prior to the test and therefore varied across baboons. Presentation conditions and response modalities were identical to those of the training phases. Differential reinforcement was given.

\section{Results}

Figure 2 shows the percentage of correct responses for each baboon and training session. There were noticeable variations in learning speed in Training Phase 1. All the baboons except one (i.e., B03) performed poorly at the beginning of Training Phase 1 but rapidly reached the learning criterion in subsequent training sessions. Altogether, the baboons needed from two to eight training sessions to reach criterion in the first training phase (see Figure 2). B03 and B11 were the two fastest animals to learn; one (i.e., B03) was trained with upright faces, and the other (i.e., B11) was trained with inverted faces. Since the other animals were also trained with either upright (B07) or upside-down (B08) pictures, there is no evidence of any systematic relation between facial orientation and length of training.

Noticeable also in Figure 2 is the performance decrement between the final session of Phase 1 and the initial session of Phase 2. Two-tailed chi-square tests were computed to test the significance of this effect. For B03, B07, and B08, there was a significant performance decline between the last session of Training Phase 1 and the first session of Phase 2 [B03, $\chi^{2}(1, N=160)=7.9 ; \mathrm{B} 07, \chi^{2}$ $(1, N=160)=4$; B08, $\chi^{2}(1, N=160)=7.1 ;$ all $p \mathrm{~s}<$ $.05]$. No subsequent comparisons between the final ses- 



Figure 2. Percentage of correct responses for each baboon in each session of the five training phases.

sion of one training phase and the first session of the next phase revealed such significant differences. The significant performance decrement between Training Phases 1 and 2 shows that these stimulus pairs were perceptually different for the baboons.

Figure 3 presents the individual number of correct responses out of 12 in each test condition. Binomial twotailed tests comparing the individual numbers of correct and incorrect responses revealed that each baboon performed at chance level in the different condition (all $p \mathrm{~s}>$ .05 ) but performed significantly above chance in the same condition (all $p s<.05$ ). These two results show an effect of stimulus rotation, which occurred irrespective of the orientation of the training pictures.

\section{Discussion}

Two main conclusions derive from Experiment 1. First, during the training phases, no clear-cut differences emerged between the experimental groups. Indeed, the baboons belonging to the control group (trained with upside-down faces) did not depart from the animals of the canonical group in terms of learning speed. This effect seems at odds with previous results showing that humans discriminate upright human and ape faces more easily than upsidedown ones (e.g., Phelps \& Roberts, 1994). This latter re- sult is also inconsistent with the observation that a squirrel monkey (Samiri sciureus) showed faster acquisition with upright human and ape faces than with inverted ones (Phelps \& Roberts, 1994). It should be noted, however, that they only tested 1 monkey in each of their experiments.

The second main conclusion that emerges from our experiment is that rotation of the training stimuli by $180^{\circ}$ had the same adverse effect in the two experimental groups: In both groups, rotation disrupted animals' abilities to discriminate those stimuli (see the Notes section for comparative human data ${ }^{1}$ ), suggesting that the effect of a $180^{\circ}$ rotation was unrelated to the initial orientation of the training faces. This latter result questions the possibility that animals processed the pictorial stimuli as equivalent to the real faces they depict. Before discussing these findings in depth, we will present a second experiment that was aimed at confirming these initial observations.

\section{EXPERIMENT 2}

The main innovation of Experiment 2 was the use of a priming procedure to test the effect of stimulus inversion on face discrimination in nonhuman primates. That procedure was adopted in the context of this research because it is well suited to reveal some forms of covert (im- 
Figure 3. For each baboon, individual number of correct responses out of 12 in Experiment 1 as a function of trial type (same vs. different).

plicit) recognition of the human individuals in the pictures. In this experiment, a picture of a face was presented as a prime stimulus prior to the display of the target face. Primes could show either the same individual as the target face or a different one. Moreover, the prime face was presented either under the same orientation as the target or in a different orientation. The baboons from the control and canonical groups were requested to give a go or a no-go response on the basis of the target face only; no explicit response had to be given on the basis of the priming stimulus. We hypothesized that any special encoding of stimulus orientation should give rise to shorter response times when the prime and the target had identical orientations than when they had different orientations. Although priming procedures have been used to study face processing in humans (e.g., Brunas, Young, \& Ellis, 1990), this experiment is, to the best of our knowledge, the first to investigate priming effects on face processing with nonhuman species.

\section{Method}

Subjects, Apparatus, and Stimuli. The baboons and apparatus were identical to those of Experiment 1. The stimulus set comprised 36 novel pictures of the same two humans as before (18 pictures per individual). The human models were photographed following the same procedure as that in Experiment 1 .

Testing procedure. The general testing procedure was identical to the go/no-go procedure of Experiment 1, except that a prime stimulus was presented after the cursor had been placed on the starting stimulus and prior to the onset of the target face. The prime was displayed for $120 \mathrm{msec}$. Use of the fixation procedure ensured that the prime was really perceived prior to the presentation of the target face (see Wilde et al., 1994). There was a fixed time interval of $170 \mathrm{msec}$ between the offset of the prime and the onset of the target, during which the joystick was inactivated. Rewards were delivered contingent on responses to the target stimuli only, irrespective of the prime stimuli.

Each baboon received eight test sessions of 48 trials (total, 384 trials). Sessions comprised eight testing conditions, each being re- peated six times. The eight testing conditions resulted from the orthogonal manipulation of the following three experimental factors: (1) the identity of the individual depicted on the target (defining the go or the no-go trials); (2) the identity of the individual depicted on the prime, either the same individual as for the target (the Same-I condition) or the different one (the Dif-I condition); (3) the orientation of the prime, either the same (the Same-O condition) or different from the target (the Dif- $O$ condition). The baboons always perceived the target in the same orientation as that during the training phases of Experiment 1 (upright for B03 and B07, upside down for B08 and B11). The other experimental aspects of the procedure-for instance, in terms of target presentation duration or reinforcement contingencies - were identical to those of the previous experiment.

A total of 24 different pictures (12 pairs) were used as target, each being displayed once with an upright prime and once with an upside-down prime. The set of priming pictures comprised $12 \mathrm{im}$ ages different from the target pictures. Each prime was presented four times within a session, once per individual (Same-I vs. Dif-I) by orientation (upright, upside down) conditions. The order of trial presentation was randomized, with the constraint that no more than three go or no-go trials be presented in succession.

Training. To familiarize the baboons with the priming procedure, a first training phase was conducted using junk artificial shapes (e.g., polygons) as priming stimuli and the two keepers' faces as target stimuli. Once performance was above $90 \%$ correct over two consecutive sessions, animals proceeded to training on the same task, but with faces as primes. This training phase consisted of only three sessions of 48 trials per animal, because they met or exceeded $90 \%$ correct in the first session. This training phase involved eight novel pairs of faces, four as prime and four as target.

\section{Results}

B03, B07, and B08 performed at $97.6 \%, 95.5 \%$, and 93.9\% correct, on average. Such high performance levels prevented further analyses of scores, in view of possible ceiling effects. B11 performed at $81.8 \%$ correct, on average. Inspection of its data revealed a strong bias for nogo responses when the prime and the target showed the same individual - in particular, when they were both upside down $(40.8 \%$ correct). B11 was discarded from data 
analyses, because this bias invalidated possible comparisons across conditions.

The analysis of response times focused on correct go trials. Because there was some variability in response speed-for instance, owing to response anticipations or inattention-mean response times were calculated for each baboon and experimental condition, and any individual data point exceeding two standard deviations from that mean was omitted for statistical analysis. This procedure removed only $5.3 \%$ of the data set.

We first verified whether there were response time differences between the Same-I and the Dif-I conditions when the prime and the target faces had the same orientation. Correct response times were significantly shorter in the Same-I than in the Dif-I condition (see Figure 4A). This effect emerged for B03 $[t(88)=2.09, p<.04]$ and $\mathrm{B} 07[t(83)=2.04, p<.05]$, for which faces were upright, and for $\mathrm{B} 08[t(87)=4.14, p<.001]$, for which faces were upside down. Thus, there was no clear-cut difference between the results of the baboons trained with upright and with inverted faces.

A second analysis compared response times in the Same-O and Dif-O conditions when the prime and the target represented the same keeper. For the 3 baboons, response times were shorter in the Same-O condition than in the Dif-O condition (see Figure 4B). Two-tailed $t$ tests on correct response times revealed this difference

\section{A}

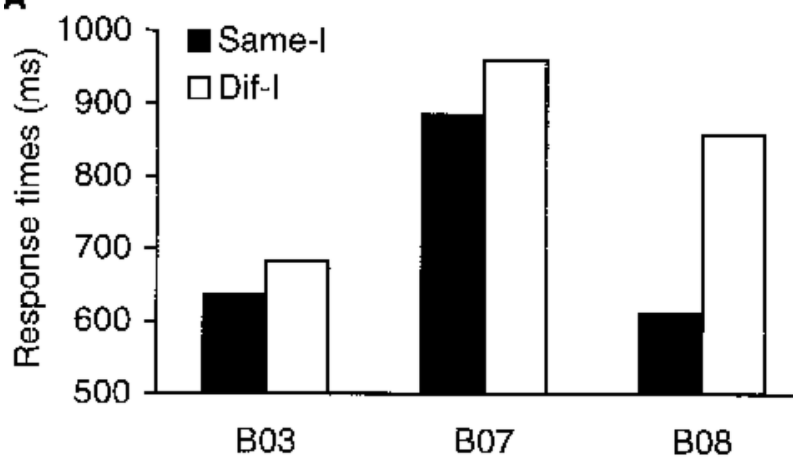

B

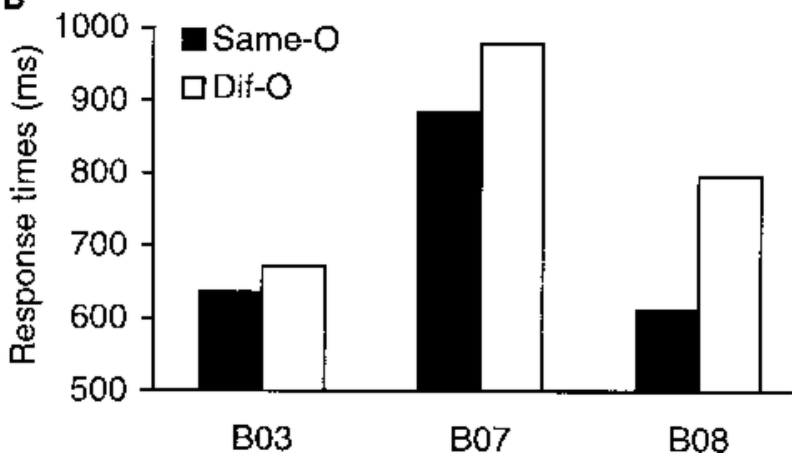

Figure 4. Individual mean response times in Experiment 2, depending on the identity of the individuals depicted on the prime and target stimuli (panel $A$ ) and the orientation of the prime relative to the target face (panel $B$ ). to be significant for B03 $[t(88)=2.17, p<.05]$ and B07 $[t(83)=2.14, p<.05]$, which perceived the target faces upright, and for B08 $[t(87)=4.63, p<.001]$, which perceived them upside down. Consistent with the results of Experiment 1, these findings reveal an effect of stimuli rotation irrespective of the orientation (upright or upside down) of the target during initial training and testing.

Two-tailed $t$ tests also compared response times when the prime and the target stimuli showed different keepers with the same orientation (Dif-I/Same-O condition) with response times when the prime showed the same individual as the target, but in an inverted orientation (Same-I/ Dif-O). None of the response time differences emerged as significant (all $p \mathrm{~s}>.10$ ), showing that priming effects were neither stronger nor weaker in these two conditions.

\section{Discussion}

The two groups of subjects showed similar effects of face inversion on discrimination speed. In these two groups, response times were shorter when the prime and the target had identical orientations than when they had different orientations. One limitation of this experiment is that the primes were always facial stimuli. A neutral condition is thus missing to determine whether the primes facilitated performance in the same trials or, rather, altered performance on different trials. It is noticeable, however, that the presentation of the faces in their canonical orientation offered no measurable advantage to the baboons (see note 2 for comparative human results). This result is, at first glance, surprising because our baboons, which are terrestrial animals, have in their everyday life a greater expertise with upright real human faces than with upside-down ones.

To account for this phenomenon, it can be proposed that the baboons did not process those pictures as representations of real faces but, instead, as nonmeaningful visual stimuli. This conclusion accords with the results obtained in Experiment 1, showing that the canonical group did not differ from the control group in terms of learning speed. It also accords with some previous findings from the animal literature on picture perception and categorization, which showed that nonhuman primates and birds occasionally rely on the analysis of local features to discriminate complex realistic images (D'Amato \& van Sant, 1988; van Hamme, Wasserman, \& Biederman, 1992). Considering this hypothesis, the aim of the following two experiments (Experiments 3 and 4) was to provide additional insight into the processing of facial stimuli by the baboons and to identify any salient cues on which the animals might be relying in our face discrimination task.

\section{EXPERIMENT 3}

In this experiment, monkeys discriminated pictures of faces in a go/no-go discrimination task. The original pictures were used as targets in some trials, whereas some facial features were manipulated in selective ways in other trials. Performance with the altered pictures was an- 
alyzed to identify which aspect(s) of the pictures controlled discrimination behavior.

\section{Method}

Subjects, Apparatus, and Stimuli. The subjects and apparatus were the same as those in the previous two experiments. The original stimulus set consisted of two pictures of faces, each representing one of the two animal keepers. The test set comprised the same two faces as the original set, which were altered in several ways. Because previous studies had demonstrated that local features might be referred to by animals for picture discrimination (e.g., D'Amato \& van Sant, 1988), we questioned whether or not such features could have controlled baboons' behaviors in our experiments. For that purpose, we identified a list of picture characteristics that appeared to be salient enough (from a human perspective at least) to allow discrimination of the pictures of the two keepers. These features were the presence of skin blemishes, eye reflections, variations in the shape of the nostrils or eyebrows, and variations in the overall contour of the faces. In order to verify whether these features really controlled discrimination, six pairs of stimuli were made from the original two digitized pictures, which were modified by (1) erasing the eye reflections, (2) erasing skin blemishes, (3) changing the shape of the eyebrows to have them similar in the two pictures, (4) changing the shape of the nostrils to have it similar in the two pictures, and (5) including the internal parts of the two faces within an oval (see Figure 5). The sixth pair was simultaneously altered for eye reflection, skin blemishes, brows, nostrils, and contour. It is important to note that, at least from a human perspective, the two faces were still highly discriminable, whatever the transformation applied to them. Moreover, a rapid informal experiment revealed that people knowing those two persons still easily identified the two models on the altered pictures.

Testing procedure. The procedure was the same as that in Experiment 1 . Each baboon received 12 sessions of 48 trials (576 trials total), during which the target faces were presented either upright (canonical group: B03 and B07) or upside down (control group: B08 and B11). The 12 test images of the six pairs were presented

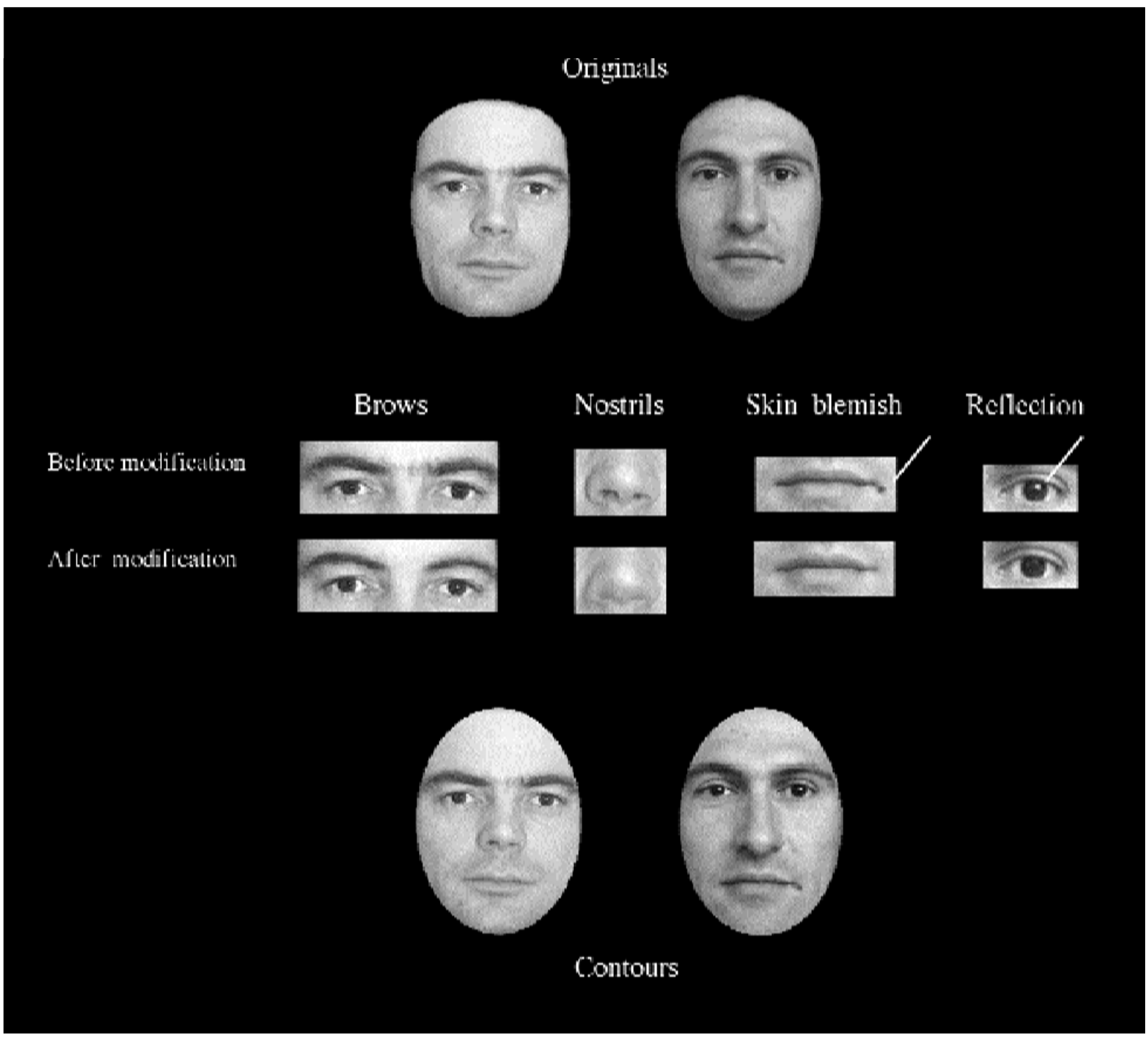

Figure 5. Original pair of pictures used in Experiment 3 (top), and modifications introduced in the test pictures (bottom). 
Table 1

Number of Errors per Baboon Over the 24 Trials of Each Test Condition

\begin{tabular}{ccccccc}
\hline Subject & Blemishes & Eyes & Brows & Nostrils & Contours & All \\
\hline B03 & $0^{*}$ & $0^{*}$ & $1^{*}$ & $0^{*}$ & 11 & 12 \\
B07 & $0^{*}$ & $1^{*}$ & $0^{*}$ & $1^{*}$ & 12 & 10 \\
B08 & $2^{*}$ & $2^{*}$ & $2^{*}$ & $0^{*}$ & 8 & 10 \\
B11 & $0^{*}$ & $0^{*}$ & $0^{*}$ & $1^{*}$ & 13 & 11 \\
\hline
\end{tabular}

Note-"Blemishes," "Eyes," "Brows," "Nostrils," and "Contours" respectively refer to the alteration of the skin blemishes, eye reflections, shape of the eyebrows, shape of the nostrils, and facial contours. "All" indicates the simultaneous alteration of the latter five facial features. *Performance significantly above chance as inferred by a two-tailed binomial test $(p<.05)$.

once within a session. Differential reinforcement was given. The other trials involved presentation of the original pictures (18 times each per session), which were intermixed with the altered pictures.

\section{Results}

Response times were not analyzed because of the small number of test trials per condition. Individual performance remained maximal with the original faces (more than $99 \%$ correct). Performance with the test faces varied across test conditions (see Table 1). Performance was significantly above chance $(97.4 \%$ correct, on average) when picture alterations concerned the skin blemishes, the eye reflection, the shape of the eyebrows, and the shape of the nostrils (two-tailed binomial tests, all $p s<$ $.05)$. However, performance remained at chance level when the internal parts of the face were placed within an oval (55.8\% correct, on average) or when all the alterations were simultaneously applied to the faces $(55.2 \%$ correct, on average). The results thus point to variations in facial contour as the main (if not the only) discriminative cue used by the baboons.

\section{Discussion}

Experiment 3 showed that an alteration of facial contours disrupted performance, thus indicating that contour variations were important cues for the baboons. By contrast, manipulations of the internal features of the faces had no clear-cut effects. The fact that the monkeys could not discriminate the faces when presented within an oval shape is consistent with the hypothesis that they did not process the pictures as representations of human faces and did not process the identities of the human individuals represented in the pictures. Remember that an informal experiment with humans revealed that the two animal keepers remained discriminable by human observers after alteration. This fact suggests that some differences exist in the processing of these pictures by humans and baboons (see notes 1 and 2 for convergent observations). In the case of the baboons, Experiment 3 revealed that discrimination performance was, in a very large part, based on the analysis of facial contours, a feature that is a weaker predictor of facial identity than the internal part of the face. Experiment 4 tested in a more direct way the relative saliency for baboons of the exter- nal (i.e., contour) and internal parts of the face in our face discrimination task.

\section{EXPERIMENT 4}

Experiment 4 assessed whether contour-related cues were more salient for the baboons than the internal parts of the faces. For that purpose, the baboons had to respond to chimeric faces, which were created by inserting the internal parts of the face of one model within the contour of the face of the other model (see Figure 6). A conflict was thus introduced between internal facial features and contour information.

\section{Method}

Subjects, Apparatus, Stimuli, and Procedure. The baboons, apparatus, and general procedure (i.e., go/no-go task) were the same as those in Experiment 3. The stimulus set comprised two pictures of the original faces that were taken by following the same procedure as that in Experiment 1, as well as the two chimeric faces shown in Figure 6. Each chimeric face was made by including the internal region of the face of each model within the facial contour of the other model.

Each baboon received three sessions of 96 trials each. Test sessions consisted of 88 trials involving the original faces (44 go and 44 no-go trials) and 8 trials involving the chimeric faces (4 trials with each face). As before, faces (originals and chimerics) were presented upright for B03 and B07 and upside down for B08 and B11. Because there was no correct answer to chimeric faces, presentation of the chimeric was reinforced at a .5 probability. Trials with the original faces were reinforced each time a correct go or no-go response was given. The other aspects of the procedure were the same as those in Experiment 3.

\section{Results and Discussion}

The number of go and no-go responses to chimeric faces indicated whether these stimuli were classified on the basis of their contour or their internal parts. Based on a binomial two-tailed test $(p<.05), \mathrm{B} 03$ and B08 had a significant preference for contour-based responses (in 22 of the 24 trials for both subjects). Note that the faces were displayed upright for B03 and upside down for B08, suggesting that this response strategy did not depend on the orientation of the faces. B07 showed a slight preference for contour-based responses (15 of 24), but that preference was not significant ( $p>.05)$. By contrast, the remaining baboon (B11) exhibited a significant bias for responding on the basis of the internal features (in 20 of 24 trials; $p<.05$ ). In sum, for at least 2 baboons, the contour was a more salient discriminative cue than the internal facial features. The other 2 baboons showed either no preference for either face property (B07) or responses based on the internal region of the face (B11).

It might be proposed that this last baboon was capable of recognizing the individual humans on the pictures and relied on the identity of these individuals to discriminate the facial stimuli. However, the fact that this same baboon could not discriminate the facial stimuli when they were shown within an oval (Experiment 3) makes that hypothesis unlikely. Indeed, use of individual identities to discriminate the pictures in Experiment 4 should have 


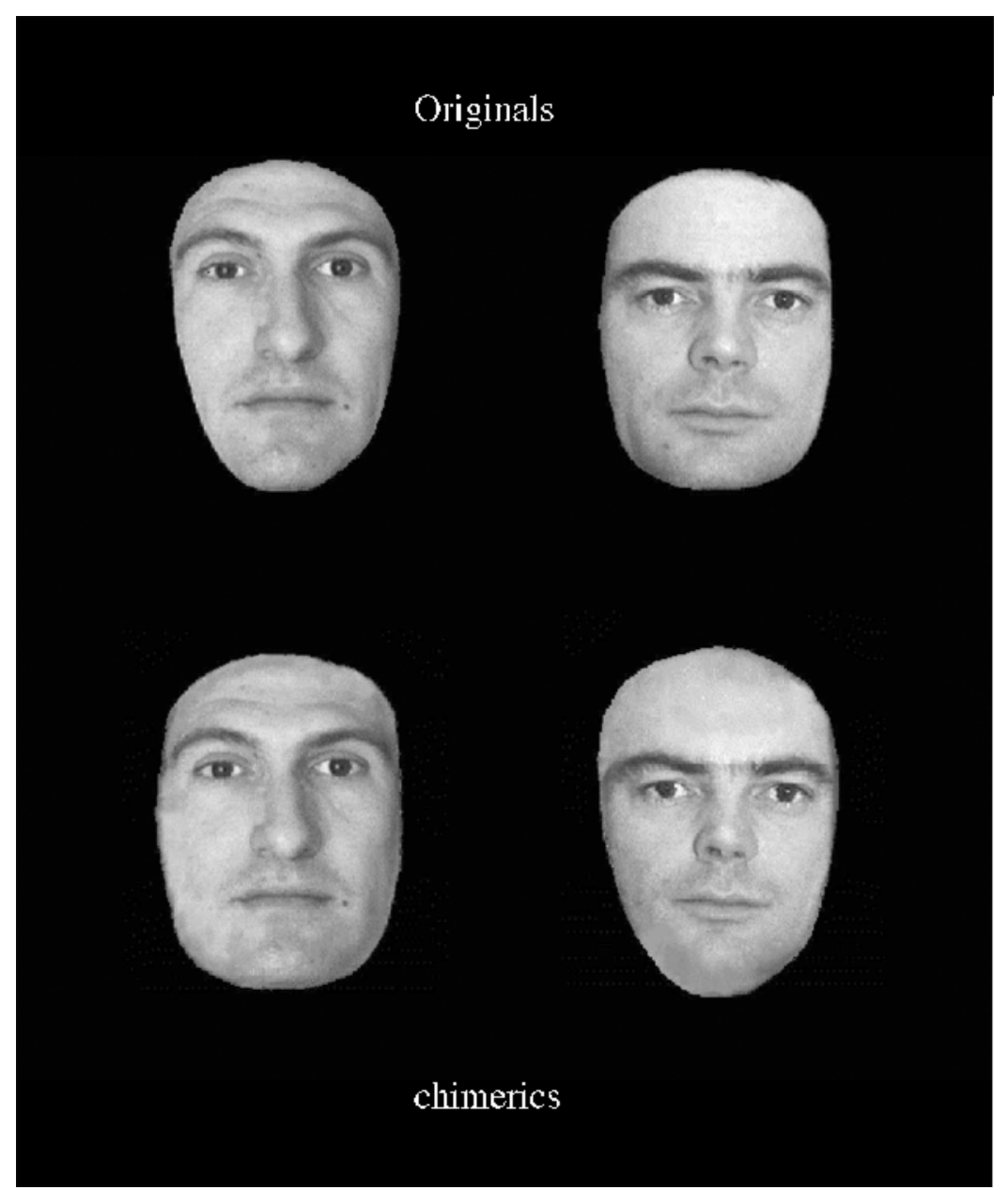

Figure 6. Original and chimeric pictures of Experiment 4. Chimerics were created by inserting the internal facial parts of one keeper's face into the contour of the other keeper's face, and vice versa.

given a high level of performance in both Experiments 3 and 4 . After inspection of the test stimuli of Experiment 3 and of the chimerical stimuli of Experiment 4, we propose that another feature could have been considered by B11 to discriminate the pictures. In particular, in comparison with the original pictures, placing the faces within the ovals almost equated the size of the foreheads of the two individuals (see Figure 5), whereas forehead size remained almost unchanged for chimeric faces (see Figure 6). Thus, a consideration of that particular feature could explain why B 11 showed a decline of performance in the transfer test using the oval faces but classified the chimeric faces on the basis of their internal parts.

\section{GENERAL DISCUSSION}

This series of experiments questions the issue of picture perception and the mechanisms underlying the effect of stimulus rotation in nonhuman primates. Considering the issue of picture perception, some aspects of the literature suggest that nonhuman primates process pho- tographs as representations of real objects or real individuals. For instance, chimpanzees display a differential pattern of heart rate responses after viewing pictures of familiar or unfamiliar chimpanzees (Boysen \& Berntson, 1986), suggesting that chimpanzees perceive the identity of individuals in the pictures. Evidence that baboons (Catland \& Judge, 1991) look longer at slides of conspecifics also suggests representational qualities of the pictures for these animals.

In contrast to the above studies, other reports have indicated that pictures of natural living or nonliving objects are not systematically perceived by animals (including monkeys) as representations of real objects but are sometimes processed as nonrepresentative patterns. Such evidence derives from studies on picture-object equivalence and concept formation. For instance, in a study on picture-object equivalence, baboons were unable to transfer performance on a go/no-go task involving discrimination of pairs of real objects to pictures of the same objects (Martin-Malivel, 1998). In another study on concept formation, stumptailed monkeys (Macaca 
arctoides) initially trained to sort pictures on a human/ nonhuman conceptual basis exhibited poor immediate transfer of performance when they were presented with novel pictures of the same concepts (Schrier, Angarella, $\&$ Povar, 1984). Similarly, cebus monkeys (Cebus apella) relied on absolute local cues (a reddish coloration) to discriminate full frontal pictures of humans from pictures without humans (D'Amato \& van Sant, 1988). Furthermore, the same animals showed poor transfer of performance to close-up views of faces. In macaques also, Jitsumori and Matsuzawa (1991) reported a similar lack of transfer from full views of humans to faces.

In the context of our experiment, interestingly, those baboons trained with upright faces (canonical group) did not reach learning criterion in Experiment 1 faster than those trained with upside-down faces (control group): In the two groups, there was one fast- and one slow-learning animal. This result contrasts with the data from humans, showing better discrimination and greater memorization of upright than of upside-down faces (e.g., Yin, 1969). Also striking is the fact that the canonical and the control groups behaved similarly in the test trials of Experiments 1 and 2. Thus, rotation of the facial pictures similarly altered discrimination in the two groups of baboons (in Experiment 1). In both groups, moreover, priming effects occurred when the prime and the target pictures had the same orientation, irrespective of the orientation of the training pictures (in Experiment 2).

Although Experiments 1 and 2 suggest that facial pictures were not processed by the baboons as representations of real faces, they provide little information as to the stimulus dimensions controlling discrimination. That problem was alleviated in Experiment 3 by selectively altering some aspects of the faces. In that case, the baboons' performance was disrupted when facial contours were altered. This finding rules out the possibility that baboons learn to discriminate facial identities on the basis of the internal regions of the faces. Finally, Experiment 4 provided a clear-cut demonstration in 2 baboons (B03 and $\mathrm{B} 08$ ) that contour variations controlled discrimination behavior much more than did variations of the internal features and that a particulate internal feature might have controlled discrimination behavior in a 3rd baboon (B11). From these findings, it might be argued that facial processing in baboons differs from that in humans, both in a lack of superiority for upright faces and in greater attention to facial contour than to more informative internal facial features. Although our experiments cannot completely rule out this hypothesis, a more likely explanation for the present results is that the baboons did not process the pictures as representations of faces and did not recognize the correspondence between the pictures and the keepers they represented.

The findings of the present research are not trivial and have both methodological and theoretical implications. On the methodological side, this study questions the adequacy of testing procedures involving picture displays for inferring the processing of three-dimensional objects in the real word. Clearly stated, the present study does not rule out the possibility that monkeys or other primate species might process pictures as realistic stimuli. However, it does question the validity of picture presentation procedures when adequate controls have not been proposed to ascertain that pictures are processed as equivalent to the real objects (or individuals). On a more theoretical side, there is a long-lasting debate in the animal conditioning and learning literature on whether or not discrimination performance in categorization tasks reflects feature analysis or template learning. Schematically, feature analysis refers to the fact that a single feature or a constellation of various meaningless features, such as line patterns or blobs of colors, gains control over the animals' discriminatory behavior (e.g., Lea \& Ryan, 1990). Alternatively, template learning implies the processing of the stimulus as a whole (e.g., Roitblat \& von Fersen, 1992), instead of a decomposition of some of its featural aspects. The results from Experiments 3 and 4 are more in line with the feature analysis than with the template learning theory, because these two experiments identified a single salient feature (the contour) as the main cue controlling discrimination. Because the baboons apparently processed facial pictures irrespective of what they represented (from a human perspective), it remains undetermined at this point whether the feature analysis theory would similarly account for the processing of real faces.

The question remains of why rotation of the training stimuli $180^{\circ}$ affected the baboons' discrimination in Experiment 1 . The literature on humans has reported effects of stimulus rotation with stimuli other than faces (e.g., houses or airplanes, Yin, 1969) —in particular, in tasks involving repeated presentations of mono-oriented objects (dot patterns, Tanaka \& Farah, 1991; discrimination of dog pictures by dog experts, Diamond \& Carey, 1986). In line with these experiments, the present research suggests that our baboons encoded the orientation of the training pictures, as humans apparently do with familiar mono-oriented objects.

From a different perspective, Experiments 1-4 indicate that observing the effects of stimulus inversion in a face discrimination task involving pictures demonstrates neither that the faces are processed as equivalent to the real faces nor that the effect of stimulus inversion involves specialized face systems. Further comparative studies should complement the investigation of face inversion effects with a real attempt to identify the stimulus-processing mode adopted by the animals in the test conditions.

\section{REFERENCES}

Boysen, S. T., \& Berntson, G. G. (1986). Cardiac correlates of individual recognition in the chimpanzee (Pan troglodytes). Journal of Comparative Psychology, 100, 321-324.

BRUCE, C. (1982). Face recognition by monkeys: Absence of an inversion effect. Neuropsychologia, 20, 515-522.

Brunas, J., Young, A. W., \& Ellis, A. W. (1990). Repetition priming from incomplete faces: Evidence for part to whole completion. British Journal of Psychology, 81, 43-56.

Catland, D. K., \& Judge, P. G. (1991). Visual categories of Macaca 
fuscata and Papio hamadryas. In A. Ehara, T. Kimura, O. Takenaka, \& M. Iwamoto (Eds.), Primatology today (pp. 289-292). Amsterdam: Elsevier.

D' Амato, M. R, \& van SANT, P. (1988). The person concept in monkeys (Cebus apella). Journal of Experimental Psychology: Animal Behavior Processes, 14, 43-55.

Deruelle, C., \& FAgOT, J. (1997). Hemispheric lateralization and global precedence effects in the processing of visual stimuli by humans and baboons. Laterality, 2, 233-246.

DiAmond, R., \& CAREY, S. (1986). Why faces are and are not special: An effect of expertise. Journal of Experimental Psychology: General, 115, 107-117.

DitTRICH, W. (1990). Representation of faces in longtailed macaques (Macaca fascicularis). Ethology, 85, 265-278.

FAGOT, J. (ED.) (2000). Picture perception in animals. Hove, U.K.: Psychology Press.

FAgot, J., \& Deruelle, C. (1997). Processing of global and local visual information and hemispheric specialization in humans (Homo sapiens) and baboons (Papio papio). Journal of Experimental Psychology: Human Perception \& Performance, 23, 429-442.

Fagot, J., Martin-Malivel, J., \& Dépy, D. (1999). What is the evidence for equivalence between objects and pictures in birds and nonhuman primates? Current Psychology of Cognition, 18, 923-949.

Farah, M. J., Wilson, K. D., Drain, H. M., \& Tanaka, J. W. (1998). What is "special" about face perception? Psychological Review, 105, 482-498.

Hasselmo, M. E., Rolls, E. T., Baylis, G. C., \& Nalwa, V. (1989). Object-centered encoding by face-selective neurons in the cortex in the superior temporal sulcus of the monkey. Experimental Brain Research, 75, 417-429.

Huber, L. (1999). Generic Perception: Open-ended categorization of natural classes. Current Psychology of Cognition, 18, 845-887.

Jitsumori, M., \& MatsuZaWA, T. (1991). Picture perception in monkeys and pigeons: Transfer of rightside-up versus upside-down discrimination of photographic objects across conceptual categories. Primates, 32, 473-482.

LEA, S. E. G., \& RYAn, C. M. E. (1990). Unnatural concepts and theory of concept discrimination in birds. In M. L. Commons, R. H. Herrnstein, S. M. Kosslyn, \& D. B. Mumford (Eds.), Quantitative analyses of behavior: Vol. 8. Behavioral approaches to pattern recognition and concept formation (pp. 165-185). Hillsdale, NJ: Erlbaum.

Martin-Malivel, J. (1998). Existe-t-il une équivalence entre l'objet et sa représentation photographique chez le babouin (Papio papio)? Primatologie, 1, 249-268.

Overman, W. H., \& Doty, R. W. (1982). Hemispheric specialization displayed by man but not macaques for analysis of faces. Neuropsychologia, 20, 113-128.

PArr, L. A., Dove, T., \& Hopkins, W. D. (1998). Why faces may be special: Evidence of the inversion effect in chimpanzees. Journal of Cognitive Neuroscience, 10, 615-622.

Pascalis, O., Petit, O., Kim, J. H., \& Campbell, R. (1999). Picture perception in primates: The case of face perception. Current Psychology of Cognition, 18, 889-921.

Perrett, D. I., Smith, P. A. J., Potter, D. D., Mistlin, A. J., Head, A. S., Milner, A. D., \& Jeeves, M. A. (1984). Neurons responsive to faces in the temporal cortex: Studies of functional organization, sensitivity to identity and relation to perception. Human Neurobiology, 3, 197-208.

Phelps, M. T., \& Roberts, W. A. (1994). Memory for pictures of upright and inverted primate faces in humans, squirrel monkeys and pigeons. Journal of Comparative Psychology, 108, 114-125.

Roitblat, H. L., \& von Fersen, L. (1992). Comparative cognition: Representations and processes in learning and memory. Annual Review of Psychology, 43, 671-710.

Rosenfeld, S. A., \& VAN Hoesen, G. W. (1979). Face recognition in the rhesus monkey. Neuropsychologia, 17, 503-509.

Schrier, A. M., Angarella, R., \& Povar, M. L. (1984). Studies of concept formation by stumptailed monkeys: Concepts humans, monkeys, and letter A. Journal of Experimental Psychology: Animal Behavior Processes, 10, 564-584.

SWARTZ, K. B. (1982). Species discrimination in infant pigtail macaques with pictorial stimuli. Developmental Psychobiology, 16, 219-231.
TANAKA, J. W., \& FARAH, M. J. (1991). Second-order relational properties and the inversion effect: Testing a theory of face perception. Perception \& Psychophysics, 50, 367-372.

Tomonaga, M., Itakura, S., \& Matsuzawa, T. (1993). Superiority of conspecific faces and reduced inversion effect in face perception by a chimpanzee. Folia Primatologica, 61, 110-114.

VAlentine, T. (1988). Upside-down faces: A review of the effect of inversion upon face recognition. British Journal of Psychology, 79, 471-491.

Van Hamme, L. J., Wasserman, E. A., \& Biederman, I. (1992). Discrimination of contour-deleted images by pigeons. Journal of Experimental Psychology: Animal Behavior Processes, 18, 387-399.

Vermeire, B., \& Hamilton, C. (1998). Inversion effect for faces in split-brain monkeys. Neuropsychologia, 36, 1003-1014.

Wilde, J., VAuclair, J., \& FAgot, J. (1994). Eye movements in baboons performing a matching-to-sample task presented in a divided field format. Behavioural Brain Research, 63, 61-70.

Wright A. A., \& Roberts, W. A. (1996). Monkey and human face perception: Inversion effects for human faces but not for monkey faces or scenes. Journal of Cognitive Neuroscience, 8, 278-290.

YIN, R. K. (1969). Looking at upside-down faces. Journal of Experimental Psychology, 81, 141-145.

\section{NOTES}

1. Because, to our knowledge, the procedure employed in Experiment 1 has never been proposed for humans, we ran a control experiment with 4 human subjects in order to verify whether they would behave like the baboons in our test or would show stronger effects of stimulus rotation when trained with upright than with upside-down faces. Each subject initially received five training sessions, similar to those of the baboons, in which faces were presented upright for two subjects (canonical group) and upside down for the other two (control group). After training, the subjects received two test sessions of 24 trials, in which the test pictures were presented either upright (for half of the trials) or upside down (for the other half). Only response times were considered for the analysis, because of the extremely low number of error trials (two errors in total). The results of a group (canonical, control) $\times$ test condition (upright, upside down) analysis of variance indicated a significant two-way interaction $[F(1,2)=20.2, p<.05]$; stimulus rotation significantly hampered discrimination for those subjects trained with upright faces (mean difference $=49 \mathrm{msec}$, Tukey test, $p<.05$ ) but had no significant effect for the other 2 subjects (mean difference $=$ $6 \mathrm{msec}$, Tukey test, $p>.1$ ). This result is in sharp contrast with those of the baboons. It demonstrates that the effect of stimulus rotation was asymmetrical in humans, that effect being stronger for those subjects trained with upright faces than for the other subjects.

2. For comparative purposes, we ran a control experiment in order to verify how humans would behave in that experiment. The same 4 humans as those in the control test of Experiment 1 (see note 1) were thus trained and tested, using the exact same procedure as with the baboons. Their response times were then submitted to an analysis of variance in which the factors were group (canonical, control), identity (prime and target showed the same individual, Same-I; prime and target showed different individuals, Dif-I), and orientation (prime and target had the same orientation, Same-O; prime and target had different orientations, Dif$\mathrm{O})$. None of the main effects emerged as significant. The three-way group $\times$ identity $\times$ orientation interaction was, however, significant $[F(1,2)=43.0, p<.03]$. Post hoc analyses (Tukey tests, $p<.05$ ) revealed that for both groups, response times were faster in the Same-I than in the Dif-I condition, when the prime and the targets had the same orientation. However, the effect of orientation differed for the two groups on Same-I trials. For the canonical group, the Same-O condition gave rise to shorter response times than did the Dif-O condition. For the control group, there was no significant difference between Same-O and Dif-O trials. These results confirm that the effect of orientation is asymmetrical in humans, that effect being stronger for the canonical group than for the control group.

(Manuscript received November 12, 1999; revision accepted for publication August 8, 2000.) 